\title{
EDITORIAL
}

\section{The Changing World Of Military Health Care}

\author{
I Jenkins
}

I count it a particular honour to be invited to deliver the 2004 AMSUS international keynote address and I am very grateful to the programme organisers for giving me this opportunity to take stock of the present status of military medicine and, at this very fitting time, to consider the topic of Military Health Care in a Changing World.

There has certainly been enormous change in the world throughout the past twenty years during which we have moved from the static stand-off of two great military alliances namely NATO and the Warsaw Pact each with large-scale combat medical provision based largely in the then-divided Europe to campaigns to combat the threats of global terror and to respond to regional crises necessitating rapid-reaction forces supported by lean and flexible medical facilities. Today's circumstances demand smaller more flexible medical services able to deliver a greater medical capability of a higher quality. These medical services need to be expeditionary in nature with many held at high readiness. (Our very recent and extremely short notice deployment to the Ivory Coast to evacuate British and other entitled foreign nationals is an example of the sort of operation that we may all face in the future).

The key defence medical outputs, however, remain the same: trained and deployable operational medical capability; and the maintenance of fit service personnel by effective health promotion, education and injury prevention and by the provision of prompt and effective diagnosis, treatment and rehabilitation.

I would like to map out how the United Kingdom's Defence Medical Services have met these challenges by reconfiguring the provision of healthcare both at home and on deployed operations. I will cover not only the nature of the operations and the political influences that have dictated our size and shape, but the changes in relationships with civilian colleagues and other Nations with whom we now work so closely. Associated with this we have had to respond to new national clinical quality initiatives demanding new standards of Clinical Governance in peace-time and on operations and to firmly establish Military Medicine on a sound scientific and evidence base.
I will describe how we provide healthcare at home to the non-deployed military population as that is critical to being able to deploy a fighting force that is fit for task, and I will cover the changing face of medical support on deployed operations and how we are relying more on multinational cooperation to burden-share (for example through organisations such as NATO, the EU or through bilateral agreements and coalitions).

Firstly, however, I will set the scene by briefly describing the recent history of the UK medical services which I inherited and which will be familiar to some of you.

During the Cold war we contemplated a potentially large-scale war with the concomitant threat of nuclear devastation affecting much of Europe and possibly North America. Large armies composing NATO and the Warsaw Pact faced each other in Europe across the North German Plains. In Europe the United Kingdom had extensive medical support in the form of mobile Field Hospitals and up to five Rear-based hospitals. In 1994 the UK still had eleven Military Hospitals, seven in the British Isles, two in Germany with a Royal Naval Hospital in Gibraltar and a Royal Air Force hospital in Cyprus.

Unexpectedly, however, in 1982 Great Britain suddenly found itself in an expeditionary conflict 8000 miles from home in the Falkland Islands in the South Atlantic. We were not well configured for such an event and we learned many lessons including the need to deploy smaller units more rapidly than before and how to deploy for operations which had not necessarily been planned or exercised. This brief, but intense, conflict highlighted some capability deficits leading to reviews of the principles of battle surgery, the management of major burns and of the importance of recognising and managing the consequences of battle stress. In addition we had to reconsider the implications of longrange logistics and strategic aeromedical evacuation.

Following this brief expeditionary conflict the UK revisited its defence medical strategy in order to revitalise our expeditionary capability whilst maintaining our enduring contribution to the defence of Western
States, Denver,

Colorado 
Europe.

On the 9th of November 1989 the Berlin Wall fell and with the de-escalation of the Soviet threat there was a great military and political opportunity to reduce our defence budget and to reap the benefit of the socalled 'peace-dividend' leading to our 'Options for Change' initiative.

However, in 1990 we were thrust again into another unlikely crisis when we joined the coalition to expel the occupying Iraqi army from Kuwait. The UK mobilised 5410 medical personnel to support some 40000 British Servicemen deployed to Operation GRANBY, as we called it, providing 49 surgical teams and 1850 beds including one 600 bed Field Hospital. Fortunately the numbers of casualties incurred by the coalition under utilised these facilities and prompted a debate whether such a medical capability was necessary for the future. Operation GRANBY provided a major lesson on the need for adequate force preparation and record keeping. It highlighted the need to deploy a robust record system, preferably electronic, to follow the individual and which could be easily incorporated into main medical records back at home. We also saw the surfacing of post-conflict syndromes, which produced a montage of lessons surrounding force preparation especially in immunisation and psychological issues, their recognition and appropriate treatment.

Once again when the dust from the first Gulf war settled, and with the Warsaw Pact dissolved there was a further opportunity to reduce defence expenditure which prompted a 'Front-line First' initiative in the United Kingdom. This resulted in significant cuts in the size of our medical services, a fundamental reorganisation and major changes in the way that British military healthcare was delivered.

The most profound changes involved secondary healthcare and reduced our last three homeland Military Hospitals to one (the Royal Naval Hospital Haslar near Portsmouth which became tri-Service). The other Military Hospitals were replaced by five Ministry of Defence Hospital Units (MDHUs). These MDHUs are staffed by military medical personnel in existing National Health Service Hospitals throughout the Country in areas of military concentration. In addition organisational changes were made in the delivery of medical training, dental services and medical supply. Shortly after these changes were imposed our newly elected Government undertook a Strategic Defence Review which recognised that we were incompletely configured to respond to the demands of modern warfare and which sought to restore the numbers of regular and reserve medical personnel. It was subsequently decided to close the sole surviving Military Hospital and to open a centre of academic military medicine - the Royal Centre for Defence Medicine in Birmingham.

We had by now moved into an era where virtually all our secondary care medical personnel worked on a day-to-day basis in National Health Service hospitals alongside civilian colleagues and where the secondary care treatment of military patients was performed in civilian units.

The advantages are that Defence Medical Services practitioners now have access to a broader clinical specialty mix and can more easily retain the breadth of skills that are required for military medical operations.

However, it is sometimes difficult to maintain military ethos and culture in our practitioners who are solely employed in a civilian environment and NHS hospital managers undoubtedly feel deprived when significant numbers of their embedded military staff are mobilised. In Military Hospitals UK military patients had little or no waiting time for admission for elective treatment. A major disadvantage of having to rely on NHS hospitals is that we now have no influence on the waiting-times for military patients to be admitted for treatment. Admission priorities are based solely on clinical urgency with no recognition of the need for Service patients to return swiftly to operational fitness or for overall operational capability. Where these criteria must be met fast-track treatment in fee-paying private hospitals has to be utilised.

Medical training and postgraduate education is the province of a Defence Agency (Defence Medical Education and Training Agency) and we are at present reconfiguring the strategic oversight and planning of all military healthcare into a new Headquarters Directorate.

We have increasingly recognised the need in recent years to encourage our executive colleagues to subscribe to sport and training methods that are less likely to cause injury whilst we have expanded considerably our rehabilitation services by opening new Regional Rehabilitation Units and expanding the delivery and research capability of our Defence Rehabilitation Centre at Headley Court.

Also, in response to operational experience, we have reconfigured our Mental Health Services to a community-based service and have closed our Military Psychiatric hospital. Service patients needing in-patient psychiatric assessment and treatment are now admitted under a contract with a UK private provider. The community service is now more focused on the needs of the deployable servicemen, reactions to hostile environments and associated domestic stressors.

The strategy for British Defence Medicine in the $21 \mathrm{st}$ Century is based on the identification and recognition of medical 
threats which means studying the pathophysiology and devising appropriate therapies for the types of illness and injury induced by existing, new and novel weapon systems including biological and chemical agents. This can only be successful with a greater access to Medical Intelligence and by developing an expertise in analysing such information. This identifies where capability gaps exist against defined threats and where Research efforts and resources are best directed, invested in and prioritised and will generate a much stronger foundation on which to develop Medical Doctrine, clinical regimes and protocols. This, in turn, determines Medical Training and drives the Medical Equipment Programme. All this will better inform recruiting strategies and help to decide the specialty-mix required in the Defence Medical Services of the future. This information will inform Military Doctrine and Concepts and ensure that our manpower calculations and priorities for Defence Medicine are appropriate to the delivery of Operational Capability.

The foci for much of this work are the Royal Centre for Defence Medicine, the Institute of Naval Medicine and the RAF Centre for Aviation Medicine Henlow. (RCDM also acts as our main aeromedical reception hospital and tertiary referral centre where appropriate).

During the period of reorganisation of military healthcare in the UK, the Balkans crisis was emerging and it became apparent that in order to fully resource such an operation Multinational coalitions and groups of nations had to work together to create a capability that was truly expeditionary. The crucial issue was that of interoperability to deploy quicker and more comprehensively than we had been accustomed to. This included more rapidly deployable medical assets and I will deal with these issues separately.

Throughout operations in Bosnia, Kosovo, Afghanistan and now in Iraq, we have seen various coalitions and, within these, various configurations of multinational medical support. From the UK's perspective we have seen cooperation in the form of the Multinational Medical Unit (MMU) in Sipovo where UK, the Netherlands (NL), Canada (CA) and Iceland have worked together through a Memorandum of Understanding (MOU). The experiences of working together have matured into robust working relationships. These have formed the basis for the development of a UK/NL deployable Role 3 facility and such bilateral relationships are suitable building blocks for future MMUs.

In Kosovo, UK personnel worked within the US Role 3 at Camp Bondsteel. This arrangement highlighted that for some MMUs there may be an issue of certification of medical personnel due to national legislation and in some theatres of operation there may need to be a nominated lead Nation. These environments are ideal for embedding small capability-based medical teams from other Nations. For example, currently we have a relationship where Czech Republic surgical teams are working within the UK Role 3 in Iraq.

In Afghanistan, we have seen a transition from a few nations working together in the International Security Assistance Force (ISAF) when Germany provided a Role 3 at Camp Warehouse and the French Role 2 at Kabul International Airport, to a Multinational brigade with 25 nations and medical support being provided by Germany in conjunction with Jordan, the Netherlands, Norway, Spain and others. We also rely on Jordanian Role 3 in Sierra Leone.

One of the consistent lessons from these Multinational Medical Task Forces is that nations cannot be put together indiscriminately; coalitions need to be born out of natural groupings based on familiarity of language, culture, professional congruence and from having exercised together previously. This is particularly important for the EU and NATO with the recent expansion of both organisations and with more countries ambitious to join. There is a need to concentrate on developing interoperability with smaller groups of familiar nations particularly given the potential for very rapid deployment. Again the development of the UK/NL deployable Role 3 and our close relationships with Denmark and Norway are examples of natural pairing of culturally similar nations with an established rapport.

NATO recognises this need for greater interoperability and has reconfigured its Allied Command structure. This is epitomised by the creation of Allied Command Transformation (ACT) in Norfolk Virginia, and Allied Command Operations (ACO) within its new structure. ACT is the focus for the development of NATO doctrine and concepts. Many of you will be aware of the work being conducted on Allied Joint Publication (AJP) 4.10 upon which new member nations are looking to base their national medical doctrine. Additionally, more medical courses are being developed at the NATO school in Oberamergau for example on Major Incident Medical Management and Support (MIMMS), Medical Intelligence and for Senior Medical Staff Officers to enable us all to work together more coherently. ACO now has the demanding task of becoming an effective Operational Headquarters and generating MMUs for NATO operations. To this end they have initiated a project to produce a NATO common-funded Role 2 to be manned by a lead nation in coalition with other nations especially those who do not have their own infrastructure. This is a new 
and most exciting development and paves the way for increased commonality for medical doctrine and equipment as more nations train to use the common NATO infrastructure.

You may think that I have forgotten to mention the huge contribution of the United States. Quite on the contrary: current UK operations invariably involve planning and working hand-in-hand with US Forces. Moreover, the UK has initiatives to develop our relationship with the US Air Force, as our medical teams on deployed airbases tend to share the often limited facilities. In the past, and certainly during the recent Iraq war, US and UK medical personnel have developed local arrangements for working together. This is obviously an area where more can be achieved. Additionally, based on experience in the Balkans, we are working on a MMU Role 3 MOU for the Army cooperation group of America, Britain, Canada and Australia (ABCA). And bringing us up to date we currently have the US providing Role 3 and aeromedical evacuation for our soldiers working in support of coalition operations south of Baghdad.

Traditionally Strategic Aeromedical Evacuation (AE) is usually a national responsibility on operations. However, bilateral arrangements exist as just mentioned and the UK routinely returns other Nationals from our area of responsibility to $\mathrm{UK}$ where their own $\mathrm{AE}$ service collects them. Nevertheless, we feel that more efficient use of $\mathrm{AE}$ flights is possible and I am aware that an initiative by the European Capability Action Plan Medical Project Group (ECAP Med PG) has resulted in the European Air Group (EAG) scoping the creation of an European Union Aeromedical Evacuation Coordination Centre (EU AECC). Initially this would be to simply utilize spare capacity on existing flights or on empty return legs. Unlike the interoperability we are striving for in other areas we recognise that this capability is best exploited by sharing capacity with other nations rather than by mixed manning or unification of airframes or medical equipment.

In additional to interoperability the other direction in which we are moving is increased strategic agility. We now have the concepts of NATO Reaction Forces (NRF) and European Battle Groups (EU BGs). These high-readiness military contingents demand light, small Role 2s or 3s easily deployable by air on 30 days Notice-to-Move (NTM). This is consistent with UK lessons identified in the recent conflict in Iraq. These lessons demonstrated that 25 to 50 bed Role $3 \mathrm{~s}$ with minimum infrastructure were required in the early insertion phases that could redeploy rapidly. These facilities, however, need to be capable of expansion to larger Role 3s with enhanced equipment scales and broader specialist expertise when the battlespace becomes more defined.

I mentioned earlier the requirement to develop military healthcare based on evidence and robust science. On deployment there are national differences on how peacetime standards may be maintained and when variation from the so called 'goldstandard/best practice' is tolerable. Most nations agree, however, that we should all aspire to meet the same standards on operations as at home wherever practicable. This principle is agreed in the NATO document 'Military Committee (MC) 326/2'. Additionally, in the UK we have introduced a process of Clinical Governance following some well publicised failures in healthcare systems and rogue doctors in our National Health Service. Clinical Governance includes the requirement for ongoing audit, ensuring that personnel are appropriately trained for the roles to which they are assigned and in general demonstrating that there is a process for ever improving professional standards.

It is British policy to insist that these clinical quality standards continue where possible on all exercises and operations. This can be as simple as auditing Mass Casualty (MASCAL) plans and exercising appropriately, or extend to include the audit of such topics as infection control and medical supply. Through these processes we hope to achieve ever improving standards of healthcare on operations such as the introduction where possible of the most technologically advanced imaging techniques and telemedicine and telediagnosis. Improvement in clinical care on operations includes pre-deployment preparation and post-deployment follow up and, following the lessons we learned covering mental health that I mentioned earlier, we have introduced systems for predeployment briefings and enhanced counselling support for those returning from traumatic experiences together with access when requested to skilled advice on reintegration into normal family life.

There will, of course, be other areas for developing Defence Medicine in the 21st Century. The threat of bioterrorism is here to stay and we participate in joint civilian and military planning. Post-conflict Syndromes (so-called Gulf War Syndrome) and Post Traumatic Stress Disorder may be inevitable products of operational deployments and mitigation strategies are being devised. I believe that the anticipation of such conditions should be built into operational planning and be considered in calculating casualty rates and when forecasting the cost of deployments, whether they be war fighting, or peace enforcement/keeping. Vaccines, immunisation protocols and other medical countermeasures are being 
developed, based on existing and new science, and there may be new requirements for humanitarian and disaster relief medicine, possibly of greater magnitude as world climates change.

It is vital (for us) to anticipate and predict what challenges will emerge for Defence Medicine in the 21st Century and to establish new and appropriate research and clinical strategies. British Defence Medicine had been largely 'surgicentric', had incompletely embraced the breadth of potential threat and been suboptimally configured to react to, study or develop expertise in some of the areas of risk. It has now been revitalised to establish it on a sound scientific and evidence base whilst recognising existing and emerging weapons systems (including psychological) and developing prophylactic countermeasures and clinical protocols for treatment.

Potential enemies will change as has been witnessed in recent years and new threats will emerge from other nations, regimes, terrorists, fanatics or any combination of such. There will, certainly, be new weapons systems, conventional or otherwise, symmetric or asymmetric, generating new patterns of injury possibly not confining their devastation to a purely military population. We continue to develop new combat systems bringing with them a need to exploit new applications of physiology for survival as aerospace and below water battle spaces are further exploited. New technology will bring new vulnerability and will demand new medical countermeasures some not yet contemplated. Historically military medicine has been related to surgical trauma, but this may recede and new methods of environmental and personal protection will be needed. Indeed, it is possible that, in the future, physical fighting and consequent death and injury may become less relevant if victories could be scored by non-combatant methods such as electronic and cyber-war? What is certain, and is of particularly relevance to all volunteer forces such as ours, is that perceptions are changing. People may no longer be prepared to face the military risks and sacrifice but if they do they, and the public, will rightly expect the highest standards of clinical care and governance for their Armed Forces.

Ladies and Gentlemen in conclusion we have seen great changes over the past 20 years. Some of those changes have been the result of political influence, but most importantly, lessons learned from operations and/or peer reviewed evidence that has fashioned where we are today.

For the UK, this means a military healthcare system that is based on a core regular/full-time medical cadre in partnership with the NHS at home and an increased reliance on our medical Reserve Forces. On operations, the level of commitment requires us to work with other nations in various configurations to share the burden. But clearly our ability to deploy operational healthcare is reliant on the relationships that we all, you and I, develop between each other.

This highlights the importance of international meetings such as AMSUS to strengthen the ties between all our Nations and to promote the principle of medical support to operations. 